\title{
Nutzerzentrierung in Zeiten von Social Distancing - Evaluierung eines extracurricularen Lehrformats für Studierende der Produktentwicklung
}

Anne Wallisch, Kristin Paetzold

Nutzerzentrierung in der Produktentwicklung ist ein seit Jahrzehnten intensiv beforschtes Feld mit dem wesentlichen Ziel, Nutzeranforderungen möglichst ganzheitlich zu ermitteln und beschreiben. Damit soll den potentiellen Anwendern ein Produkt geliefert werden können, das einen tatsächlichen Bedarf bestmöglich adressiert oder entsprechende Begehrlichkeiten weckt und somit einen hohen Markterfolg verspricht. In der Praxis lässt sich dennoch beobachten, dass oftmals eine eher technische Perspektive, in welcher der Mensch irgendwie enthalten ist, den Entwicklungsprozess dominiert. Der Beitrag dient als Ergebnisbericht einer praxisbezogenen, interdisziplinären Lehrveranstaltung mit dem Ziel, bereits in der akademischen Ausbildung stärker für die Anwenderperspektive zu sensibilisieren. Die im Pilotkurs partizipativ gestaltete Lehrveranstaltung zu nutzerzentrierter Anforderungserhebung wurde im zweiten Durchlauf pandemiebedingt auf Remotelehre und virtuelle Kollaboration umgestellt. Die Effekte beider Formate werden im Beitrag unter verschiedenen Aspekten bilanziert und die Ergebnisbewertung erfolgt als Reflektion des Standardcurriculums.

Keywords: Entwurfsphase, Ingenieursausbildung, Kollaboration, Nutzerzentrierung, Produktentwicklung

\section{Einleitung}

Grundlegende Voraussetzung für das Entwerfen und Entwickeln technischer Produkte und Systeme mit hohem Anwendererlebnis ist das Verständnis für die Anwender. Die Herausforderungen, die aus verschiedenen Begriffs- und Konzeptwelten der Nutzerund der Entwicklerperspektiven resultieren, sollen insbesondere durch partizipative Entwicklungsansätze bewältigt werden. In der akademischen Ingenieursausbildung spielen diese jedoch kaum eine bedeutende Rolle. Während also Entwicklungszyklen kürzer werden, macht Nutzerzentrierung die Anforderungsanalyse merklich komplexer. Vor diesem Hintergrund scheint es durchaus plausibel und nachvollziehbar, Gestaltungsentscheidungen zugunsten technischer Machbarkeit zu treffen. Gleichzeitig 
ist bekannt, dass echte Innovation auch die Marktfähigkeit und den Bedarf einer Lösung voraussetzt (Brown: 2008). Die Frage, wie bereits in der Ausbildung für dieses Spannungsfeld sensibilisiert und Rüstzeug bereitgestellt werden kann, ist demnach keineswegs trivial: Der Mensch muss eine Rolle in der Ausbildung spielen.

Der Beitrag evaluiert ein extracurriculares Lehrprojekt für Studierende der Luft- und Raumfahrttechnik, das erstmalig im Herbstsemester 2019 mit dem Ziel, den Effekt physischer Co-Präsenz in Kollaborationssituationen mit Nutzervertretern auf Planung, Konzeption und Gestaltung erleb- und damit lernbar zu machen, angeboten wurde. 2020 wurde die Frage, wie die Präsenz von Nutzerinnen und Nutzern sowie ihrer Vertreter auch bei fehlender physischer Anwesenheit gestaltet werden kann, zentral: Bedingt durch den Ausbruch des SARS-CoV-2-Virus musste der gesamte Lehrbetrieb digitalisiert und remote umgesetzt werden.

Die nachfolgende Darstellung der Fallstudie orientiert sich im Wesentlichen am tatsächlichen methodischen Vorgehen. Um die Motivation der Autoren (und damit ihre bezüglich der Kursgestaltung getroffenen Entscheidungen) transparent zu machen, werden zunächst grundlegende Aspekte aus dem Stand der Forschung zu Nutzerzentrierung und akademischer Ausbildung in der Produktentwicklung zusammengetragen. Anschließend werden Kursdesign und Durchführung des Piloten sowie dessen Umsetzung im Remoteformat präsentiert und Schlüsselergebnisse in Form von Implikationen für die akademische Ausbildung junger Produktentwickler zusammengefasst. Der Beitrag schließt mit einem kurzen Ausblick auf weitere Forschungs- und Lehraktivitäten.

\section{Nutzerzentrierung als Paradigma der Produktentwicklung}

Der Begriff des nutzerzentrierten Gestaltens wurde bereits in den 1980er Jahren von Norman und Draper (1986) zur Beschreibung von Entwicklungsprozessen, bei denen die Bedürfnisse und Interessen der potentiellen Nutzer entscheidenden Einfluss auf den Gestaltungsprozess nehmen, geprägt. Leider sind nur wenige dieser Bedürfnisse ohne nähere Untersuchung direkt sichtbar oder zugänglich. Zentral im Sinne der Nutzerzentrierung ist folglich, die Verhaltensmuster von Menschen zu verstehen und vorherzusagen. Um einen wirklich nutzerzentrierten Gestaltungsprozess zu realisieren, ist ein entsprechendes Mindset sowie die kontinuierliche Generierung und Validierung von Nutzerinformationen während des Prozesses, bedeutend (Sanders \& Stappers: 2008). 


\section{Umsetzung in der industriellen Praxis}

In der Industrie lässt sich beobachten, dass die Ausgestaltung von Nutzerzentrierung in der Entwicklungspraxis an manchen Stellen unscharf bleibt (Wallisch, Sankowski, Krause \& Paetzold: 2019). Verschiedene Tätigkeitsbeispiele so genannter Nutzerinteraktion beschränkten sich bei genauerem Hinsehen darauf, bestehende Lösungen sporadisch zu testen, um zuvor vermutete Nutzerbedürfnisse zu verifizieren (Wallisch \& Paetzold: 2018a). In anderen Fällen erarbeiten Entwicklerteams Ideen zur nutzerfreundlichen Verbesserung kompliziert anzuwendender Produkte, ohne jedoch dabei tatsächlich mit Nutzern zu interagieren (Kimbell: 2011). Unternehmen scheint es oft an Wissen und Ressourcen zu fehlen, um das Einbeziehen der Benutzer in bestehende Arbeitsabläufe zu stärken (Carlgren, Elmqvist \& Rauth: 2016). Selbst wenn Nutzertests durchgeführt werden, geschieht dies oft mit begrenztem, zufälligem Zugang zur Nutzergruppe oder intern mit Personen, die auch Produkte entwickeln und sich auf Benutzeroberflächen statt Nutzererfahrungen konzentrieren (Kosmala, van der Marel \& Björklund: 2019).

Obwohl Entwickler im Allgemeinen die Bedeutung des Fragens und Beobachtens von Nutzerbedürfnissen bestätigen, ist eine Diskrepanz zwischen dem Bewusstsein der theoretischen Bedeutung und der praktischen Umsetzung zu beobachten (Zeisel: 2006). Diese resultiert oft aus Schwierigkeiten und Unsicherheiten, die Konstrukteure beim Sammeln, Auswählen und Verstehen von Nutzerbedürfnissen (Boztepe: 2007) empfinden. Zudem berichten Entwickler, dass sie, wenn sie mit Anwendern interagieren, oft nicht wissen, wie sie die gesammelten Informationen in den Konstruktionsprozess integrieren können: Sie brauchen spezifische Daten, die alle notwendigen Elemente in ausreichender Weise enthalten, ohne dass es zu einer Informationsüberlastung kommt (Keates \& Clarkson: 2003). Das Narrativ eher weich formulierter qualitativer Nutzerwünsche unterscheidet sich stark von der Darstellung technischer Spezifikationen, mit denen Konstrukteure zu kommunizieren und zu arbeiten gewohnt sind (Salmen: 2011).

Auf kognitiver Ebene lässt sich zwar abschätzen, wie sich verschiedene Umstände auf eine andere Person auswirken könnten, doch solange nicht tiefer in die Realität dieser Person eingetaucht wird, bleibt das Erfahrungsverständnis begrenzt. Empathie wird daher als „imaginative projection into another person's situation“ (Koskinen \& Battarbee: 2003, 45), den Versuch, die emotionalen und motivationalen Qualitäten der Benutzer zu erfassen, beschrieben. Die Empathie-Bildung in der Produktentwicklung um- 
fasst demnach eine Reihe von Aktivitäten, bei denen sich Konstrukteure vorstellen sollten, wie es für sie selbst wäre, in der Position des potentiellen Nutzers zu sein (Kouprie \& Visser: 2009).

\section{Thematisierung in der akademischen Ausbildung}

Eingang in die akademische Ausbildung finden Nutzeraspekte nur langsam, wie sich am Beispiel des Standardwerkes „Pahl/Beitz Konstruktionslehre: Methoden und Anwendung erfolgreicher Produktentwicklung" zeigt. Erstmalig enthält die jüngst von Gericke \& Bender (2021) herausgegebene neunte Auflage eigenständige (Unter-)Kapitel zu Nutzerbedürfnissen (Paetzold: 2021) sowie Nutzerzentrierter Produktentwicklung (Krzywinski \& Wölfel: 2021). Wenn es aber um nutzerzentriertes Entwerfen und Entwickeln geht, dürfen nicht nur technikgetriebene Perspektiven und Prozessmodelle vermittelt werden, sondern muss auch der Mensch, in Form theoretischer Konzepte zu wesentlichen Aspekten wie Lebensstil, Handlungsmotivation und Routinen, die für ein ganzheitliches Verständnis seines Lebenskontexts notwendig sind, sowie Forschungsinstrumente, um diese empirisch zu erschließen, eine Rolle in der Ingenieurausbildung spielen (Wallisch \& Paetzold: 2018b, 2020).

Über die technische Kompetenz hinaus erfordert die Tätigkeit als Konstrukteur die Fähigkeit, unstrukturierte Probleme anzugehen, effektiv in Teams zusammenzuarbeiten und ein hohes Maß an Anpassungsfähigkeit zu besitzen (Hora: 2017). Interdisziplinäre Kompetenz sollte insgesamt als Ausbildungsziel in den Vordergrund rücken, da diese längst zur beruflichen Praxis von Ingenieurstätigkeit gehört (Rudlof: 2018). Angesichts der nach wie vor monodisziplinären Ausrichtung vieler Ingenieursstudiengänge (Graham: 2018) überrascht es kaum, dass das Feedback aus der Industrie den Absolventen einen Mangel an praktischen Arbeitsfähigkeiten (Kind, Dybov, Buchholz \& Stark: 2019), Kreativität, Befähigung zur Anwendung von Forschungsmethoden und Kommunikation (IET: 2015) attestiert.

Ansätze wie das Karlsruher Lehrmodell der Produktentwicklung (KaLeP; Albers, Burkart, Deigendesch \& Robens: 2009) integrieren zwar durchaus zentrale Schlüsselkompetenzen, Nutzer-Empathie ist aber nicht als Kursinhalt genannt. Auf der Suche nach Methoden, mit denen Entwickler die von ihnen adressierten Nutzer in ihrer Lebenswirklichkeit verstehen können, hat sich in der Designforschung die Kombination von Gestaltung mit qualitativen Methoden zu einem eigenen Studienfeld entwickelt, das in erster Linie interpretativen Traditionen folgt (Sanders \& Stappers: 2008). Allerdings werden diese Inhalte im Bereich der Curriculumsgestaltung für die Produktentwicklung nicht häufig eingesetzt (Kelly: 2020). Zusammenfassend erkennen die Autoren ei- 
nen Lehrbedarf hinsichtlich der Relevanz der Nutzeranforderungen für die Produktentwicklung: Der Fokus sollte hier auf einer ausgewogeneren Analyse von Technologie und ihren Nutzern liegen. Die Lehre von Entwickeln als Problemlösen (Pahl: 1994) sollte um die Lehre von Nutzerforschung als Problemverständnis ergänzt werden.

\section{Forschungsansatz und Methodisches Vorgehen}

Als methodischer Ansatz zur Untersuchung der Fragestellung, wie Nutzerempathie vermittelt werden kann, dient ein exploratives Fallstudiendesign. Die Fallkonstruktion erfolgt mit der Ausarbeitung eines Lehrkonzepts, das die vorgenannten Aspekte aufgreifen und ausgleichen soll. Die qualitative Fallanalyse zielt auf induktiven Erkenntnisgewinn über die Prozesse der Empathie-Bildung, um daraus curriculare Implikationen und didaktische Werkzeuge ableiten zu können. Handlungsleitend sind folgende Forschungsfragen:

- Wie kann Studierenden ein Überblick über relevante, situations- und bedarfsgerechte Inhalte zur nutzerzentrierten Produktentwicklung vermittelt werden? (Wissen)

- Wie müssen diese Inhalte inhaltlich und medial aufbereitet sein, um ein eingängiges Verständnis zu schaffen? (Verstehen)

- Wie können diese Inhalte im kontrollierten und praxisnahen Umfeld motiviert, erlebt und ein tatsächlicher Lernerfolg im Sinne von Erfahrungsaufbau erzielt werden? (Anwenden)

Die Kursgestaltung erfolgte damit in Anlehnung an die Taxonomie von Lernzielen nach Bloom (1976). Die Kompetenz der Analyse, also das reflektierende Diskutieren verschiedener Inhalte und Methoden, soll im Rahmen des Kurses zumindest angestoßen werden. Wissen entsteht als individueller Prozess in einem spezifischen Kontext und manifestiert sich in Handlungen (North: 2011), Kompetenz wird durch praktische Anwendung übertragen (Sveiby: 1998). Dementsprechend wurde ein Praxisformat entwickelt und angekündigt:

„Ziel dieses Lehrangebots ist die Sensibilisierung für frühzeitige Interaktion mit Zielnutzern und Schulung der Teilnehmer in der praktischen Umsetzung von Nutzerzentrierung als Leitmotiv der gesamten Produktentwicklung. Im Rahmen der Veranstaltung ist die Aufgabe, ein Entwicklungsprojekt iterativ zu planen und durchzuführen, kooperativ zu lösen. Frühzeitig sollen Markthypothesen und Produktideen generiert 
und Prototypen entwickelt werden. Diese sind vor anwesenden Nutzervertretern zu präsentieren und in der direkten Interaktion mit ihnen zu testen" (Aushang).

Um möglichst rasch Feedback zum Pilotkurs zu erhalten, wurde dieser nicht direkt als eigenständiges Modul beantragt, sondern die Vorlesung "Methoden der Produktentwicklung" um eine fallbasierte Übung erweitert. Da die Lebenswelten und Erfahrungen von Ingenieursanwärtern und Senioren sehr unterschiedlich und doch vergleichsweise leicht zugänglich sind, wurde diese Konstellation bei der Formulierung der Entwicklungsaufgabe, eine Alltagsunterstützung für ältere Menschen, gewählt. Die Entwicklung einer nutzerseitig als alltagsunterstützend erlebten Lösung setzt voraus, dass die Problemanalyse nicht ausschließlich technikgetriebenen erfolgt. Die Fakultät für Luftund Raumfahrttechnik bietet hierfür traditionell wenig Raum, entsprechende Arbeitsgrundlagen sind für die Studierenden daher erst zu schaffen: Das Aufgabenverständnis sollte durch lebensweltbeschreibende Materialien aus eigenen Forschungsprojekten, die Aufgabenbearbeitung durch begleitende Coaches mit Expertenwissen zur Erhebung von Nutzerbedarfen und Prototypengestaltung sowie Zugang zu Gebrauchsmaterial unterstützt werden.

Die Einführung in zentrale Methoden und Werkzeuge zur formalen Unterstützung aller beteiligten Schritte und Aktivitäten während der Iterationen zwischen Nutzerperspektive (Problemraum) und Produktspezifikation (Lösungsraum) soll in der klassischen Form von Wissensvermittlung einer Vorlesung erfolgen. Zentrale Inhalte werden für die Übung zusammengefasst und zu Beginn der individuellen Arbeitsphasen wiederholt. Konzipiert wurden diese nach Prämissen des handlungs- bzw. problembasierten Lernens: alle Teilnehmer sollen selbst aktiv werden und durch praktische Erfahrung lernen. Jedes Team verfolgt eine für die Übung gewählte Entwicklungsidee, wobei der Fokus auf dem wirklichen Verstehen der Zielnutzer und Verifizieren des Lösungsansatzes durch Befragung, Beobachtung, Sekundäranalyse und andere (Abb. 1) liegen soll.

Die Bewertung der Entwicklungsergebnisse erfolgt, von der Prüfungsleistung entkoppelt, als Diskussion der Pitches und Prototypen des Lösungskonzepts mit anwesenden Nutzervertretern. Das erste Feedback wird mittels vorbereitetem Fragenkatalogs eingeholt, was den Zugang zu den Gedanken, Gefühlen und Emotionen der Teilnehmer erleichtern (Polyani: 1985) und einen fruchtbaren Austausch anzuregen soll. Zum Veranstaltungsende sollen die Studierenden einen Evaluationsbogen zum Lehrformat ausfüllen. Um immerhin Tendenzen für mittelfristige Effekte erfassen zu können, soll mit einigen Wochen Abstand eine offene Reflexionsrunde zum Lehrformat erfolgen. 


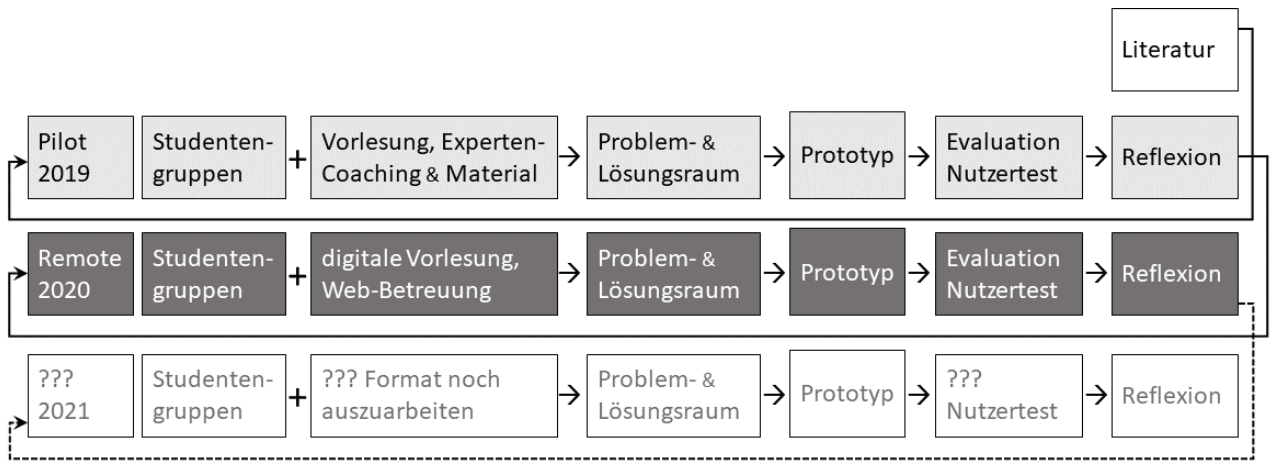

Abbildung 1: Kursdesign

\section{Durchführung und Ergebnisbewertung des Pilotkurses}

Es überrascht nicht, dass die Studierenden, obwohl hochmotiviert, den Alltag Älterer zu verbessern, sich zu Beginn des Entwurfsprozesses auf die technische Machbarkeit konzentrierten. Sie dachten in High-End-Lösungen, die zu komplex waren, um den Wunsch Älterer nach eher frugalen Systemen zu erfüllen, die diese als leichter in ihre täglichen Routinen integrierbar empfinden (Wallisch \& Paetzold: 2018c). Mithilfe der Unterstützung durch die Coaches gelang den Studenten kontinuierlich, Nutzerprobleme nicht nur technisch anzugehen, sondern auch Lösungskonzepte aus der Perspektive der anvisierten Nutzer zu bewerten.

Die Aussicht auf reelles Nutzerfeedback und Iterationshinweise durch die Anwesenheit von Nutzervertretern bei den Abschlusspräsentationen hatte einen sehr motivierenden Effekt. Das Einbeziehen von Nutzervertretern als Partner im Gestaltungsprozessprozess berichteten die Studierenden als hilfreich, den sozialen Aspekt von Produktentwicklung und dessen Einfluss auf die Lebensqualität der Menschen genauer zu erfassen, denn ein Objekt auf konkrete Bedürfnisse eines Menschen zu entwerfen, motiviert aus einem anderen Blickwinkel als das Entwerfen unter dem Aspekt technologischer Machbarkeit. Die größte Auswirkung der Nutzerinteraktion wurde in der Überarbeitung und Diskussion der Konzeptideen gesehen, die ein Gleichgewicht zwischen den Vorstellungen der Senioren und denen der Studenten, und damit klare Problemaussagen, erlaubte.

Ausgestattet mit einem traditionellen Verständnis von Prototypen als Usability-Test in den späten Phasen des Entwicklungsprozesses, erwies es sich als unerwartet mühsam, die Studierenden dafür zu begeistern, das zur Verfügung gestellte Material zu nutzen 
und einfache physische Prototypen anstelle (komplexer) digitale Modelle zu erstellen. Kontinuierliches Prototyping kann sowohl Kommunikations- als auch Erfahrungslücken zwischen Nutzern und Entwicklern minimieren (Hallgrimsson: 2012). Dieses iterative Vorgehen als Strategie nutzerzentrierten Entwickelns wird in der Ausbildung bislang nicht gut reflektiert. Das Potenzial prototypenbasierter Kommunikation, insbesondere in den sehr frühen Entwicklungsphasen, war den Studierenden nicht bewusst. Die Ausbildung sollte anregen, Prototypen als Ressource für die nutzerbezogene Anforderungserhebung zu betrachten und nicht nur für spezifische Produktiterationen. Bereits in den frühen Phasen der Produktentwicklung fördert ein gemeinsamer Makerspace die erfolgreiche Kollaboration aller Akteure (Jensen \& Steinert: 2020).

Für ihre Präsentationen bedienten die Studierenden animierte Videos, Storytelling mit Persona, Rollenspiele und klassische Vorträge zu technischen Zeichnungen. Besonders lobten die Nutzervertreter die Möglichkeit der haptischen Erfahrung eines LowFidelity-Prototypen (auf eine Übersicht der Kursergebnisse wird hier verzichtet, zu finden in Wallisch \& Paetzold: 2021). Die Studierenden waren überrascht, wie differenziert das Feedback der Nutzervertreter ausfiel und zu einer signifikanten Verbesserung ihres Entwurfs beitrug. Die Unsicherheit darüber, inwieweit die technischen Aspekte vorgeschlagener Lösungen tatsächlich verstanden wurden, erlebten beide Seiten zunächst als umständlich. Die Studierenden betonten, durch den Kurs die Bedeutung der Anforderungsanalyse für die Produktentwicklung im Allgemeinen und für die Nutzerzentrierung im Besonderen, neu verstanden zu haben.

\section{Durchführung und Ergebnisbewertung des Remotekurses}

Eine im Pilotkurses auffällige Beobachtung betrifft die Kriterien, die den Transformationsprozess von Nutzerbedürfnissen zu technischen Spezifikationen bestimmen. Auf die Frage, warum sie sich letztendlich für ein Konzept entschieden haben, antworteten die Studierenden oft: „weil es mir gefallen hat". Hier vermuten die Autoren Hinweise auf konkrete Unterstützungsbedarfe und wollten im Folgekurs explizit beobachten, inwiefern die Nutzerperspektive im Lauf des Entwicklungsprozesses von den Studierenden konsequent mitgedacht und eingebracht wird. Während der Vorbereitungen kam es SARS-CoV-2-Virus-bedingt zur landesweiten Schließung der Universitäten und der Frage, wie der Kurs virtuell und remote umzusetzen sei.

Die Kombination aus einen Webkonferenztool mit der Möglichkeit, Gruppenräume einzurichten, in denen die Studierenden jeweils kollaborativ an verschiedenen Whiteboards arbeiten konnten, war schnell gefunden. Nach der Auftaktveranstaltung stand jedoch die Frage im Raum, wie es gelingen kann, die Studierenden, die sich im Webtool 
lediglich als schwarze, stumme Fenster zu erkennen gaben, tatsächlich aktiv einzubinden und zur Empathie für die Nutzergruppe zu befähigen. Fila, Hess, Purzer \& Dringenberg (2016) bestätigen Nutzerempathie-Bildung auch für Studierende ohne direktem Nutzerkontakt, wenn diese stattdessen auf bereits für die Nutzergruppe erarbeitete Gestaltungslösungen zugreifen können. Nach den Erfahrungen des Pilotkurses können die Autoren dies nur eingeschränkt bestätigen: die Präsentation von Lösungskonzepten stimulierte eher das Denken in Lösungen als die Auseinandersetzung mit der Nutzergruppe. Auch im Remotekurs diskutierten die Arbeitsgruppen ausführlich die Lösungsskizzen, aber kaum die dahinterliegenden Alltagsherausforderungen von Senioren.

Canvas-basiertes Vorgehen beim Prototyping hat sich bereits als erfolgreiche Prozessunterstützung bewiesen (Lauff, Menold \& Wood: 2019; Hansen, Jensen, Özkil \& Martins Pacheco: 2020) und sollte für die Empathie-Bildung adaptiert werden. Unerfahrene Produktentwickler wissen selten genau, welches Wissen sie brauchen und benötigen Unterstützung bei der Formulierung von Wissensanfragen (Ahmed \& Wallace: 2004). Dementsprechend dienten die im Pilotkurs angestellten Beobachtungen als Ausgangspunkt solcher Formulierungen. Zudem sollte ein weiterer Aspekt aufgegriffen und geübt werden: Fürstenau, Langfermann, Klauser \& Born (2005) zeigen, dass Mitarbeiter ihre Projekterfahrungen nur bedingt aussagekräftig dokumentierten und die erstellten Dokumente in Textverständlichkeit und Transparenz nicht den Bedürfnissen künftiger Verwender entsprachen. Das Canvas startet daher mit Fragen zur Handlungssituation, die es zu unterstützen gilt, und wurde als MURAL-Template (Abb. 2), das durch entsprechende Farbcodierung, Design und Anordnung der Fragen die Sinnhaftigkeit gelungener Dokumentation unterstreicht, bereitgestellt.

Das Feedback der Studierenden betont dessen Wert insbesondere hinsichtlich der Strukturierung und Reflexion des eigenen Vorgehens: „am Anfang, da hatten wir alle so im Kopf wo wir so hinwollten, da hat das schon geholfen, sich selbst immer wieder auszubremsen und einen Schritt zurück zum Nutzer zu gehen“ (Student Luft- und Raumfahrttechnik), und "auch wenn man sich mit Produktentwicklung noch nie so richtig befasst hat, konnte man das Board gut und zügig abarbeiten“ (Student Mathematical Engineering).

Positiv wurde auch die Reflexion der Zugehörigkeit verschiedener Hauptfächer und damit das Zurechtfinden in heterogene Begrifflichkeiten, Theorien und Perspektiven hervorgehoben. Die Autoren vermuten die selbständig erfolgte Gruppeneinteilung der Studierenden im Pilotkurs als Grund, dass diese Anmerkung erst im Remote-format 
betont wurde, in dem die Gruppenzuteilung aus organisatorischen Gründen zufallsgeneriert via Webtool erfolgte.

Als hinderlich erwies sich der Einsatz eines Webtools hinsichtlich der Akquirierung geeigneter Nutzervertreter, die auf die nötige Technik teils weder zugreifen konnten noch wollten. Auch schien in Abwesenheit physischer Präsenz der Funke zwischen den Teilnehmern beider Seiten während der Präsentationseinheit nicht wirklich überzuspringen. Zeigten sich die Studierenden im Pilotkurs bereits unsicher darin, die angebotenen Gestaltungsmaterialien zum Prototypenbau zu gebrauchen, ging die entsprechende Motivation remote völlig verloren. Auch wenn die Lehrmaterialien exakt denjenigen aus dem Piloten entsprachen, konnte ohne Ermutigung durch die Coaches das studentische Interesse offenbar nicht hinreichend geweckt werden. Dadurch ging leider auch die Vielfältigkeit der präsentierten Prototypen verloren, und damit wiederum auch die Erfahrung, dass Studierende den Aufwand des Bauens physischer Prototypen mangels Erfahrung leicht überschätzen, wie sich im Pilotkurs beobachten ließ.

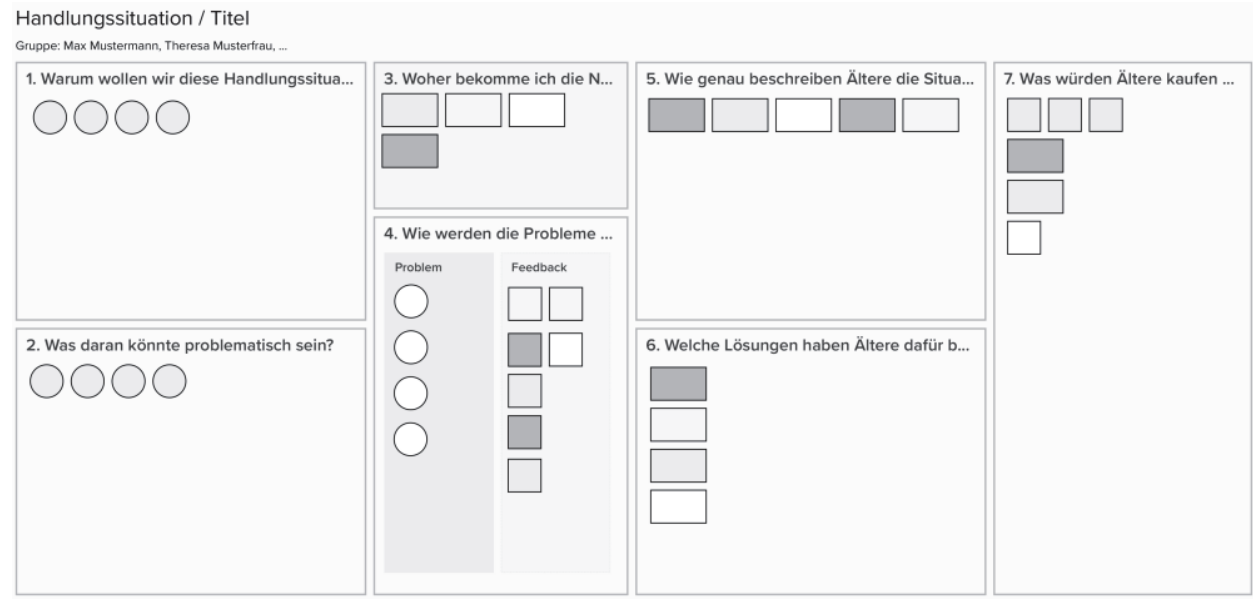

Abbildung 2: MURAL-Board: Nutzerempathie-Canvas

Konsequenter Weise bewerteten die Nutzervertreter die standardmäßig gewählte Präsentationsform animierter PowerPoint-Folien als nicht für alle Produktideen gleichermaßen geeignet, da insbesondere Dimensionen, die zum besseren Produktverständnis hätten beitragen können, nicht ohne Weiteres einzuschätzen waren. Die Coaches hätten hier unterstützen können, wurden aber kaum in Anspruch genommen. Insgesamt schienen sich die Treffen im Plenum aller Bemühungen zum Trotz durch technische Schwierigkeiten und das Fehlen mehrerer Sinnesebenen wieder mehr in Richtung 
Frontalunterricht zu bewegen. Auch konnte innerhalb des Kurses nicht genug Bindung zu den Studierenden aufgebaut werden, dass die Reflexionssitzung gut besucht worden wäre. Dadurch lässt sich an dieser Stelle nicht mit Sicherheit sagen, ob das Kursformat selbst oder eine allgemeine Müdigkeit digitaler Lehre seitens der Studierenden dafür verantwortlich war, dass nicht alle Gruppen tatsächlich ihr Ergebnis präsentierten.

Mittels Fragebogen danach befragt, wo sie den größten Ausbildungsbedarf im Studium sehen, wenn es um nutzerzentrierte Produktentwicklung geht, benannten Teilnehmer beider Kursformate: Methoden zur Validierung von Nutzerbeschreibungen, Empathie-Training, Fähigkeiten zur Kommunikation mit Laien sowie die Fähigkeiten zur Übersetzung weicher Nutzerwünsche in technische Spezifikationen. Zudem wünschten sich die Studierenden, dass der Kurs langfristiger angelegt sei, mehrere Gelegenheiten für Nutzerfeedback enthalten und den Entwicklungsprozess bis zum tatsächlichen Produkt umspannen würde.

\section{Implikationen}

Das Gestalten technischer Systeme ist nicht immer intuitiv, denn manchmal haben Entwickler anderes im Sinn als potentielle Nutzer. Das Entwerfen für neue oder bisher wenig bekannte Personengruppen kann daher eine Herausforderung sein, bietet aber zugleich die Chance, die eigenen Überzeugungen in Bezug auf Denkroutinen, Stereotypen oder den Gestaltungsprozess selbst zu reflektieren: „Man ist gewohnt, dass man an die Nutzergruppe ja meistens zum Schluss erst denkt, man ist ja ein Ingenieur und will was Supercooles machen, aber vergisst schnell mal, für wen man das macht", reflektierten teilnehmende Studierende.

Dass Problemlöser ein bestimmtes Wissen über den Realitätsbereich, in dem das Problem zu lösen ist, brauchen, ist nichts Neues (Dörner: 1979). Ein komplexer Aspekt des Gestaltens ist die Notwendigkeit, mehrere Variablen zu berücksichtigen, was Ingenieure dazu zwingt, sich mit verschiedenen Aspekten vertraut zu machen, die für das zu entwickelnde Produkt oder die Dienstleistung relevant sind. Die verantwortungsvolle Bewertung dieser Aspekte durch Bezugnahme auf verschiedene Wissensgebiete gilt als Schlüsselfähigkeit von Ingenieuren (Badke-Schaub \& Frankenberger: 2004), für die es nötig ist, neben dem entsprechenden Realitätsbereich auch die Möglichkeiten, sich innerhalb desselben zu bewegen, zu kennen. Individuelle Wahrnehmungen von Zielen können zu Beginn einer Problembearbeitung je nach Erfahrung sehr unterschiedlich sein. Um überhaupt einen Sachverhalt als Problem zu erkennen und später 
Lösungen dafür zu generieren, muss ein gewisses Ausmaß an Erfahrung mit deren Lebenswelt, Empathie für Nutzer, vorhanden sein.

Dies erfordert Forschungsaktivitäten, die das Sammeln wesentlicher Informationen, die benötigt werden, um am Ende ein geeignetes Produkt oder eine Dienstleistung zu erhalten, beinhalten, wozu die Studierenden im Kurs befähigt werden sollten. Damit liegt der Schwerpunkt nicht bei der Vermittlung von Inhalten, sondern dem individuell ausgerichteten selbstorganisierten Bearbeiten eines Themas mit dem Ziel, die Fähigkeit zu entwickeln, aus einer neuen Situation heraus eigenverantwortlich Lösungen zu entwickeln. Dieser konstruktivistische Ansatz der Lerntheorie (Reinmann-Rothmeier: 2003) sollte in der Produktentwicklung insgesamt eine größere Rolle spielen, denn dadurch können auch individuelle Bedürfnisse und Fähigkeiten, Arbeits- und Denkstile des Einzelnen besser berücksichtigt und das Anwenden von Wissen gefördert werden. Die Studierenden bestätigten den Wert der Möglichkeit, gelernte Vorlesungsinhalte, wie etwa der Anforderungsliste oder des Morphologischen Kastens, direkt und begleitet an einem Beispielfall selbst auswählen und zielgerichtet kombinieren und üben zu können. Den Nutzer zum Ausbildungsthema zu machen hat sich im präsentierten Format als notwendig und erfolgreich erwiesen. Zugleich hat sich gezeigt, dass erfolgreicher Remoteunterricht in diesem Zusammenhang etwas Anderes heißen muss, als ein Präsensformat ins Digitale zu übertragen.

\section{Schlussfolgerung und Ausblick}

Der Beitrag evaluiert die Ergebnisse eines praxisbezogenen, interdisziplinären Lehrformats Lehrveranstaltung mit dem Ziel, bereits in der akademischen Ingenieursausbildung stärker für die Anwenderperspektive zu sensibilisieren. Dieses Vorgehen unterstützt das Prinzip forschender Lehre, indem sie einen Rahmen für die Evaluierung von Lehrergebnissen schafft, sowie das Prinzip lehrender Forschung, da aktuelle Forschungsergebnisse direkt zu aktuellen Lehrinhalten führen. Lehre und Forschung werden stärker vernetzt und Anschlussstellen für die Praxis geschaffen. Das Verstehen potentieller Nutzer ist ein erfolgskritischer Faktor bei der Entwicklung neuer Produkte, von der Anforderungserhebung bis zur Markteinführung. Die Befähigung zu empathischem Verstehen sollte daher ein zentrales Lernziel in der Ingenieurausbildung werden, so wie die Annäherung von Studierenden an verschiedene Nutzergruppen eine akademische Lehraufgabe.

Zusammenfassend lässt sich festhalten, dass der direkte Kontakt mit Nutzervertretern sich positiv sowohl auf die Motivation als auch das Nutzerverständnis der Studierenden auswirkte. Dabei spielt die physische Wahrnehmung des anderen eine große Rolle. 
Dieser ,Kontakt' mit der Lebenswelt einer Nutzergruppe kann allerdings auch indirekt erfolgen, wie die Autoren aus früheren Projekten und der Auswertung von Beobachtungsvideos sowie Nutzertagebüchern wissen. Auch van Rijn, Visser, Stappers \& Özakar (2011) beschreiben, dass Videos ein immenses Potenzial zur Ausbildung von Nutzerempathie besitzen. Einen in diesem Zusammenhang interessanten Ansatz präsentiert Makki (2020), indem er die Pause-Predict-Ponder (PPP)-Methode und das dazugehörige digitalen Tool, das Ogan, Aleven \& Jones (2008) entwickelten, um Schülern eine interaktive Lernumgebung für den Erwerb interkultureller Kompetenzen zu schaffen, zur Empathie-Bildung für die Konstruktion anpasst. Die Methode beruht auf den Phasen Pause, Vorhersage und Nachdenken: Schüler bekommen Filmszenen zu sehen und sollen den Weitergang der Szene in den Pausen selbst beschreiben und ihre Antwort reflektieren. Makki $(2020,39)$ passt dieses Vorgehen für Studierende im Feld Design an, ein Ansatz, den die Autoren gelungen finden, um die physische Distanz zur Nutzergruppe in Remoteprojekten abzufangen.

Für Studierende dürfte diese Form des Unterrichts eine willkommene Ablenkung zu (vertonten) Lehrfolien bieten, und auch für die industrielle Praxis, in der ein Nutzerzugang aus verschiedensten Gründen nicht direkt möglich ist, wird erhebliches Potential gesehen. Nach wie vor sehen die Autoren Erklärungsbedarf dafür, wie sich die Transformation von empathisch-verstehenden Einblicken in Lebenswelten und Bedürfnisse potentieller Nutzer in eine für den Produktentwicklungsprozess verwertbare Form der Anforderungsbeschreibung vollzieht. Insbesondere die Unterscheidung zwischen kognitiver und affektiver Empathie sowie die Bedeutung der verschiedenen Komponenten von Empathie für die Produktentwicklung (Surma-aho, Chen, Hölttä-Otto \& Yang: 2019) verdienen mehr Aufmerksamkeit. Diese zu verstehen und erklären zu können ist grundlegend, um methodische Unterstützungsangebote zu entwickeln, mit Studierenden zu testen und schließlich in die Praxis spielen zu können. Entlang dieses Vorgehens erfolgt gegenwärtig die Weiterentwicklung des Nutzerempathie-Canvas.

\section{Literaturverzeichnis}

Ahmed, S. \& Wallace, K. (2004): Understanding the knowledge needs of novice designers in the aerospace industry. In: Design Studies, 25 (2), 155-173.

Albers, A, Burkardt, N, Deigendesch, T \& Robens, G (2009): Das Karlsruher Lehrmodell für Produktentwicklung (KaLeP) als Beispiel zur ganzheitlichen Integration von Projektarbeit in die universitäre Lehre. In: Linke, H.J. (Hrsg.): Tagungsband zum 1. Darmstädter Ingenieurkongress Bau und Umwelt, 4.-15. September 2009, Darmstadt: TU Darmstadt.

Badke-Schaub, P. \& Frankenberger, E. (2004): Management kritischer Situationen: Produktentwicklung erfolgreich gestalten. Engineering online library. Berlin: Springer. 
Bloom, B.S (Hrsg.), Engelhart, M, Furst, E, Hill, W \& Krathwohl, D (1976): Taxonomie von Lernzielen im kognitiven Bereich. Weinheim: Beltz.

Boztepe, S. (2007): Towards a framework of product development for global markets: a user-value-based approach. In: Design Studies, 28(5), 513-533.

Brown, T. (2009): Change By Design. United States: HarperCollins Publishers.

Carlgren, L, Elmqvist, M \& Rauth, I (2016): Exploring the use of design thinking in large organizations: Towards a research agenda. In: Swedish Design Research Journal, 11(1): 55-63.

Dörner, D. (1979): Problemlösen als Informationsverarbeitung. 2. Aufl., Kohlhammer-Standards Psychologie, Studientext: Teilgebiet Denkpsychologie. Stuttgart: Kohlhammer.

Fila, N.D, Hess J.L, Purzer, S \& Dringenberg, E (2016): Engineering Students' Utilization of Empathy during a Non-Immersive Conceptual Design Task. In: International Journal of Engineering Education, 32(3), 1336-1348.

Fürstenau, B, Langfermann, J, Klauser, F \& Born, V (2005): Erfahrungswissen sichern und aufbereiten - Zur effizienten Gestaltung von Wissensmanagementprozessen bei der BMW AG im Projekt „Werksaufbau Leipzig“. In: Ferstl O.K, Sinz E.J, Eckert S, Isselhorst T (Hrsg.): Wirtschaftsinformatik 2005, Heidelberg: Physica-Verlag.

Gericke, K. \& Bender, B. (Hrsg.) (2021): Pahl/Beitz Konstruktionslehre - Methoden und Anwendung erfolgreicher Produktentwicklung. Berlin: Springer Vieweg.

Graham, R. (2018): Global state of the art in engineering education. Cambridge (USA): Massachusetts Institute of Technology. Hallgrimsson, B. (2012): Prototyping and Modelmaking for Product Design. London: Laurence King Publishing.

Hansen, C, Jensen, L, Özkil, A, \& Martins Pacheco, N (2020): Fostering Prototyping Mindsets in Novice Designers with the Prototyping Planner. In: Proceedings of the Design Society: DESIGN Conference, 1, 1725-1734.

Hora M.T. (2017): Beyond the Skills Gap, National Association of Colleges and Employers, https://www.naceweb.org/career-readiness/trends-and-predictions/beyond-the-skills-gap/ veröffentlicht 2017, abgerufen am 10.01.2020.

IET, Institute of Engineering and Technology (2015): Skills and Demand in Industry Survey 2015, Institution of Engineering and Technology. https://www.theiet.org/impact-society/factfiles/education-factfiles/iet-skills-survey/iet-skills-survey-2015/, veröffentlicht 2015, abgerufen am 14.01.2020.

Jensen, M.B. \& Steinert, M. (2020): User research enabled by makerspaces: bringing functionality to classical experience prototypes. In: Artificial intelligence for engineering design analysis and manufacturing, 1-12.

Keates, S. \& Clarkson, P.J. (2003): Countering design exclusion: bridging the gap between usability and accessibility. In: Universal Access in the Information Society, 2(3), 215-225.

Kelly, P.T. (2020): Empathy Through Inquiry: The Weaving of (Post) Qualitative Inquiry into Design, Dissertation. Ann Arbor: ProQuest. Kimbell, L. (2011): Rethinking design thinking: Part I. In: Design and Culture, 3(3), 285-306.

Kind, S, Dybov, A, Buchholz, C \& Stark, R (2019): Application of industrial methods on engineering education. In: Proceedings of the 21st International Conference on Engineering and Product Design Education. E\&PDE 2019, 12-13 September, University of Strathclyde, Glasgow, 1-6. 
Koskinen, I. \& Battarbee, K. (2003): Introduction to user experience and empathic design. In: I. Koskinen, I, Mattelmäki, T \& Battarbee, K (Hrsg.): Empathic Design: User Experience in Product Design, 37-50, Helsinki: IT Press.

Kosmala, M, van der Marel, F \& Björklund, T (2019): Interpretations of Design Thinking Across a Large Organization'. In: Proceedings of the 22nd International Conference on Engineering Design (ICED19), 5-8 August, Delft, the Netherlands.

Kouprie, M. \& Visser, F.S. (2009): A framework for empathy in design: Stepping into and out of the user's life. In: Journal of Engineering Design, 20(5), 437-448.

Krzywinski, J. \& Wölfel, C. (2021): Industriedesign und Nutzerzentrierte Produktentwicklung. In: Gericke, K. \& Bender, B. (Hrsg.): Pahl/Beitz Konstruktionslehre - Methoden und Anwendung erfolgreicher Produktentwicklung, 684-704, Berlin: Springer Vieweg.

Lauff, C, Menold, J, \& Wood, K.L (2019): Prototyping Canvas: Design Tool for Planning Purposeful Prototypes. In: Proceedings of the 22nd International Conference on Engineering Design (ICED19), 5-8 August 2019, Delft, the Netherlands.

Makki, A. (2020): Design Method to Enhance Empathy for User-Centered Design: Improving the Imagination of the User Experience. Carleton University Ottawa: Unpublished thesis.

Norman, D.A. \& Draper, S.W. (1986): User-Centered System Design: New Perspectives on Human-Computer Interaction. In: Journal of Educational Computing Research, 3(1), pp. 129-134.

North, K. (2011): Wissensorientierte Unternehmensführung: Wertschöpfung durch Wissen. 5. Aufl., Wiesbaden: Gabler Verlag.

Ogan, A, Aleven, V, \& Jones, C (2008): Pause, predict, and ponder: use of narrative videos to improve cultural discussion and learning. In: Proceedings of the SIGCHI Conference on Human Factors in Computing Systems (CHI 'O8), ACM, New York, NY, USA, 155-162.

Paetzold, K. (2021.): Nutzerbedürfnisse. In: Gericke, K. \& Bender, B. (Hrsg.): Pahl/Beitz Konstruktionslehre - Methoden und Anwendung erfolgreicher Produktentwicklung, 137-167, Berlin: Springer Vieweg.

Pahl, G. (1994): Psychologische und pädagogische Fragen beim methodischen Konstruieren: Ergebnisse des Ladenburger Diskurses vom Mai 1992 bis Oktober 1993. Köln: Verl. TÜV Rheinland.

Polyani, M. (1985): Implizites Wissen. 1. Aufl., Suhrkamp-Taschenbuch Wissenschaft; 543. Frankfurt am Main: Suhrkamp.

Reinmann-Rothmeier, G. (2003): Didaktische Innovation durch Blended Learning: Leitlinien anhand eines Beispiels aus der Hochschule. 1. Aufl., Huber Psychologie Praxis: Lernen mit Neuen Medien. Bern: Huber.

Rudlof, C. (2018): Neue Curricula braucht die Hochschule - Ingenieurinnen zur Arbeitsgestaltung befähigen. In: Weidner, R. \& Karafillidis, A. (Hrsg.): Dritte Transdisziplinäre Konferenz „, Technische Unterstützungssysteme, die die Menschen wirklich wollen, Hamburg 2018, Konferenzband, 19-24, Hamburg: Helmut-Schmitt-Universität.

Salmen, J.P.S. (2001): US accessibility codes and standards. In: W.F. Preiser \& E. Ostroff (Hrsg.): Universal design handbook, 1211-1218, New York: McGraw-Hill.

Sanders, E.B.-N. \& Stappers, P.J. (2008): Co-creation and the new landscapes of design. In: Co-Design, 4(1), 5-18.

Surma-aho, A, Chen, C, Hölttä-Otto, K \& Yang, M (2019): Antecedents and outcomes of designer empathy: a retrospective interview study. In: Proceedings of the ASME 2019 International Design Engineering Technical Conferences and Computers and Information in Engineering Conference (IDETC/CIE2019), 18-21 August 18-21, 2019, Anaheim, CA, USA, 1-15. 
Sveiby, K.E. (1998): Wissenskapital - das unentdeckte Vermögen: immaterielle Unternehmenswerte aufspüren, messen und steigern. Landsberg/Lech: mi, Verl. Moderne Industrie.

Van Rijn, H, Visser, F. S, Stappers, P. J, \& Özakar, A. D (2011): Achieving empathy with users: the effects of different sources of information. In: Co-Design, 7(2), 65-77.

Wallisch, A. \& Paetzold, K. (2018a): A qualitative inventory of user integration methods and their usage in product development research and practice. In: Proceedings of the 15th International Design Conference (DESIGN 2018), 20-24 May 2018, Dubrovnik, Croatia, 115-126.

Wallisch, A. \& Paetzold, K. (2018b): Framework zu Generierung zieladäquater Untersuchungsdesigns zum Erfassen von Nutzerbedarfen. In: Weidner, R. \& Karafillidis, A. (Hrsg.): Dritte Transdisziplinäre Konferenz „Technische Unterstützungssysteme, die die Menschen wirklich wollen, Hamburg 2018, Konferenzband, 151-160, Hamburg: Helmut-Schmidt-Universität.

Wallisch, A. \& Paetzold, K. (2018c): Denn sie wissen nicht, was sie wollen, Plädoyer für ein Nutzerpraxen-orientiertes Design. In: Konferenzband zum 29th Symposium on Design for X 2018, 13-24, München: Universität der Bundeswehr München.

Wallisch, A. \& Paetzold, K. (2020): Methodological foundations of user involvement research: a contribution to user-centred design theory. In: Proceedings of the 16th International Design Conference (DESIGN 2020), 26-29 October 2020, Cavtat, Croatia, $71-80$.

Wallisch, A. \& Paetzold, K. (2021): Lessons Learned, Plea for Curricularizing Design Thinking in Engineering Education. In: Eriksson, Y. (Hrsg.): Design Thinking in Various Design Context, Chapter 9, in Druck, London: Taylor \& Francis.

Wallisch, A, Sankowski, O, Krause, D \& Paetzold, K (2019): Overcoming fuzzy design practice: revealing potentials of usercentered design research and methodological concepts related to user involvement. In: Proceedings of the IEEE International Conference on Engineering. Technology and Innovation (ICE/ITMC), 17-19 June 2019, Valbonne Sophia-Antipolis, France.

Zeisel, J. (2006): Inquiry by design. New York: Norton.

\section{Kontakt}

Dipl.-Soz. Anne Wallisch, M. A.

Prof. Dr.-Ing. Kristin Paetzold

Universität der Bundeswehr München

Institut für technische Produktentwicklung

85579 Neubiberg

www.unibw.de 